\title{
Plasmon-Induced Hot Electron Amplification and Effective Charge Separation by Au Nanoparticles Sandwiched between Copper Titanium Phosphate Nanosheets, and Improved Carbon Dioxide Conversion to Methane
}

Jeong Yeon Do ${ }^{a}$, Namgyu Son ${ }^{\mathrm{a}, 1}$, Rama Krishna Chava ${ }^{\mathrm{a}}$, Kotesh Kumar Mandaria, Sadanand Pandey $^{\mathrm{b}}$, Vignesh Kumaravel ${ }^{\mathrm{c}}$, T. S. Senthil ${ }^{\mathrm{d}}$, Sang Woo Joo ${ }^{\mathrm{e}}$, Misook Kanga,

${ }^{a}$ Department of Chemistry, College of Natural Sciences, Yeungnam University, Gyeongsan, Gyeongbuk 38541, Republic of Korea

${ }^{b}$ Particulate Matter Research Center, Research Institute of Industrial Science \& Technology (RIST), 187-12, Geumho-ro, Gwangyang-si, Jeollanam-do 57801, Republic of Korea

${ }^{c}$ Centre for Precision Engineering, Materials and Manufacturing Research (PEM), Institute of Technology Sligo, Ash Lane, F91 YW50 Sligo, Ireland

${ }^{d}$ Research Department of Physics, Erode Sengunthar Engineering College, Erode 638057, India

eSchool of Mechanical Engineering, Yeungnam University, Gyeongbuk 38541, Republic of Korea

${ }^{1}$ These authors have the same contribution.

*Correspondence Author: E-mail: mskang@ynu.ac.kr

Number of pages: 17

Number of figures: 11

Number of tables: 3

Number of movies: 1 


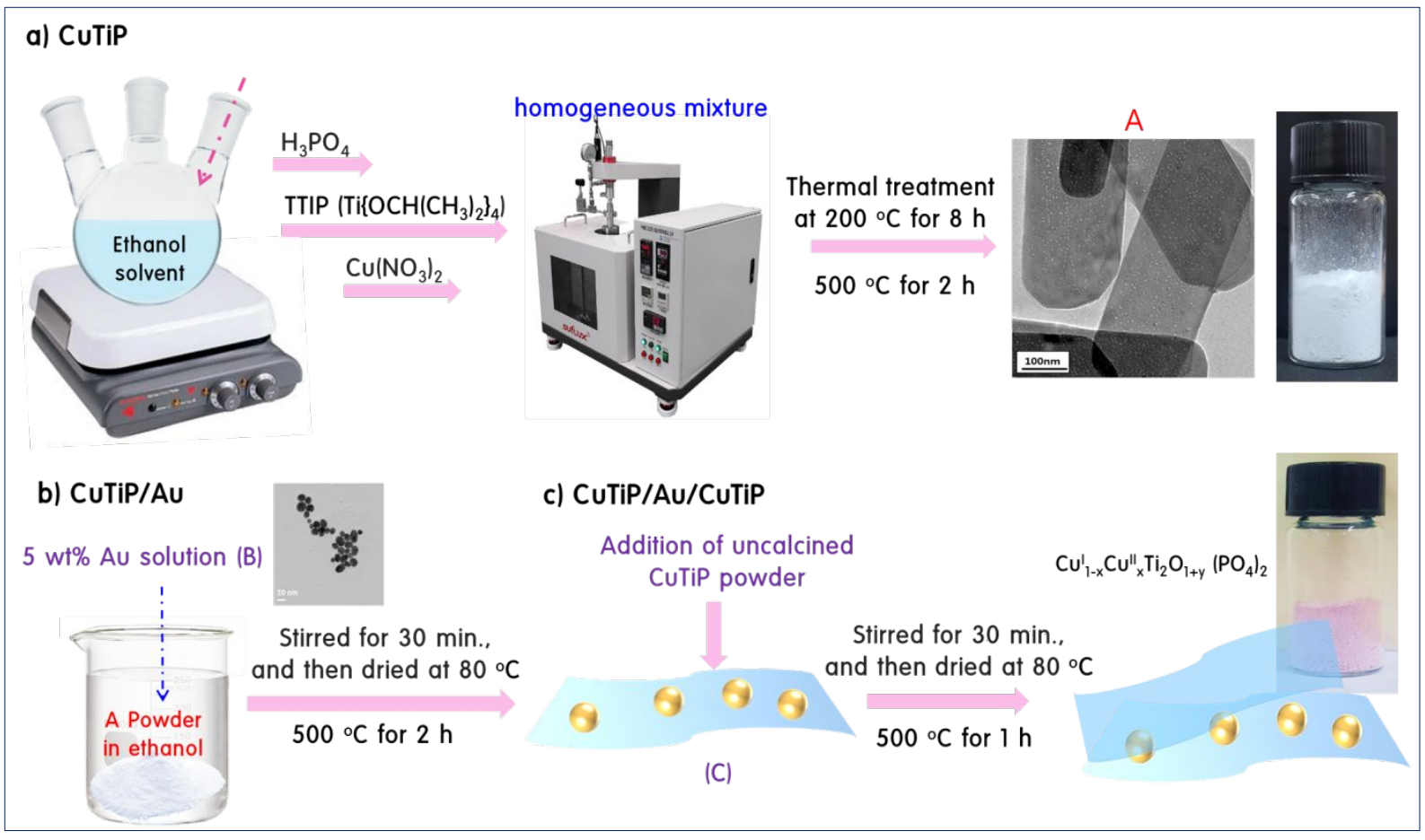

Figure S1. Synthesis process of the a) CuTiP, b) CuTiP/Au, and c) CuTiP/Au/CuTiP photocatalyst 


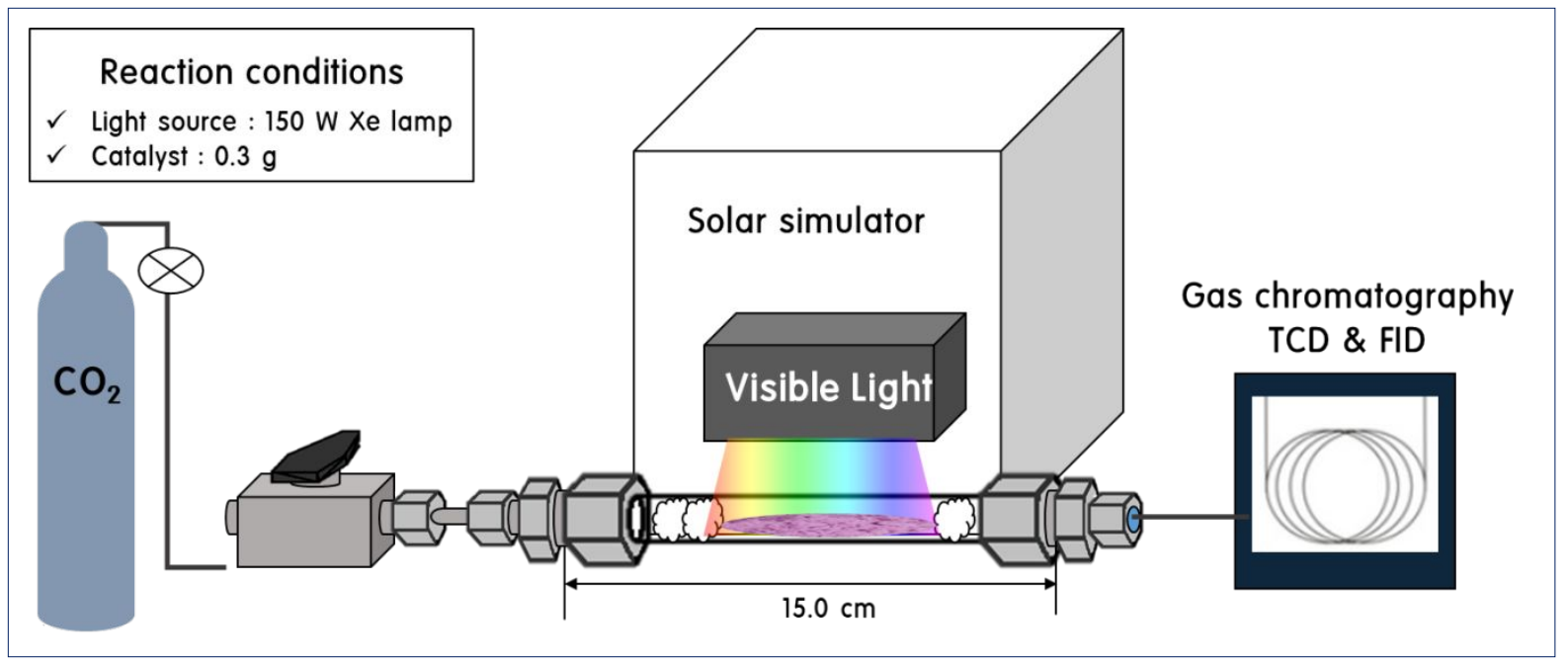

Figure S2. A gas-phase reactor schematic for $\mathrm{CO}_{2}$ photoreduction and recycling test. 


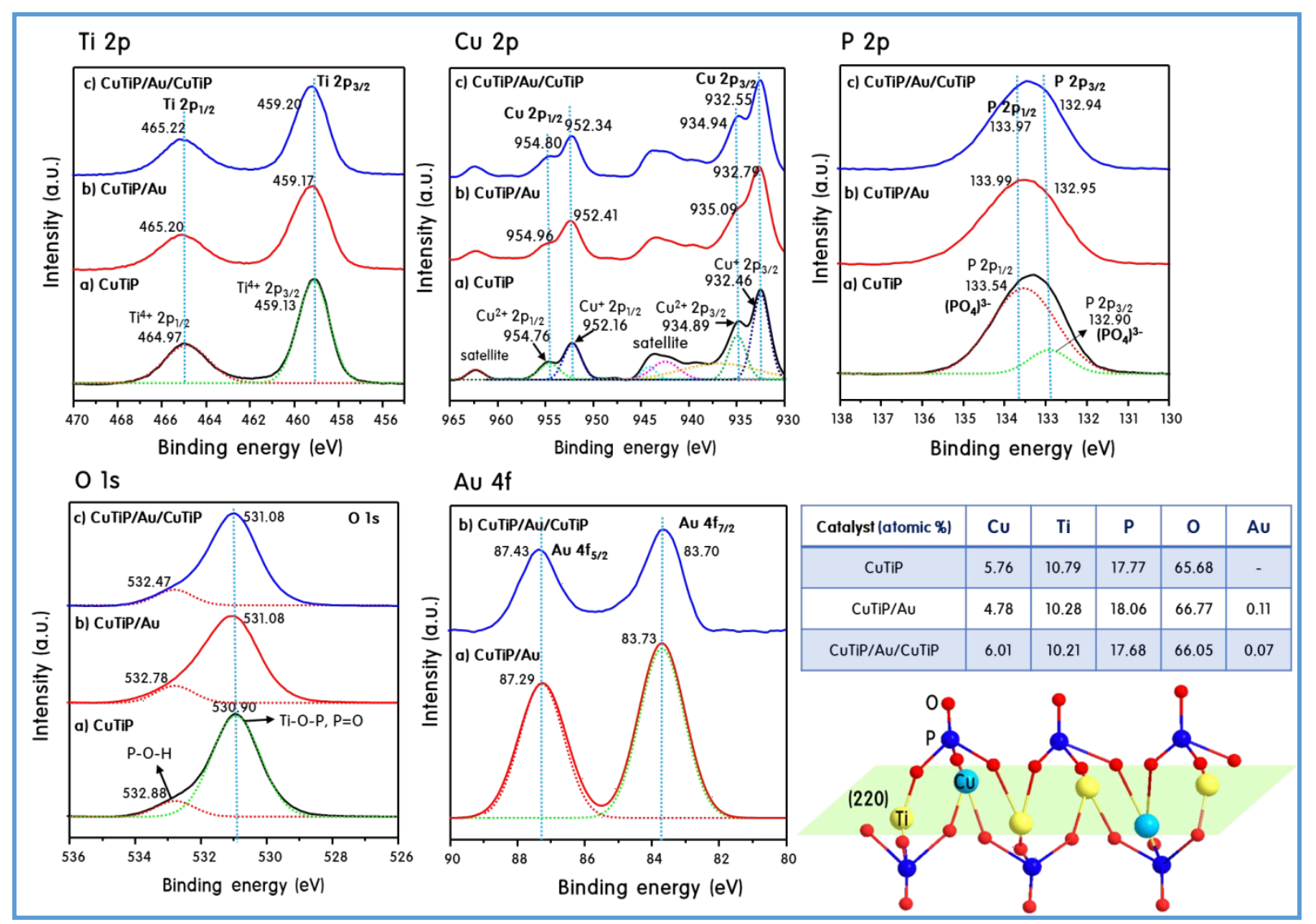

Figure S3. The XPS survey spectra and elemental analysis for a) CuTiP, b) CuTiP/Au, and c) CuTiP/Au/CuTiP. 


\begin{tabular}{lcccc}
\hline Sample & \multicolumn{4}{c}{ Measured wt.\% by ICP-AES } \\
\cline { 2 - 5 } & $\mathrm{Cu}$ & $\mathrm{Ti}$ & $\mathrm{P}$ & $\mathrm{Au}$ \\
\hline $\mathrm{CuTiP}$ & 3.43 & 13.83 & 32.62 & - \\
\hline $\mathrm{CuTiP} / \mathrm{Au}$ & 4.20 & 11.04 & 28.38 & 1.45 \\
\hline $\mathrm{CuTiP} / \mathrm{Au} / \mathrm{CuTiP}$ & 4.86 & 13.24 & 30.73 & 1.37 \\
\hline
\end{tabular}

Table S1. ICP-AES analysis of CuTiP, CuTiP/Au, and $\mathrm{CuTiP} / \mathrm{Au} / \mathrm{CuTiP}$ samples. 
A) CUTIP/Au
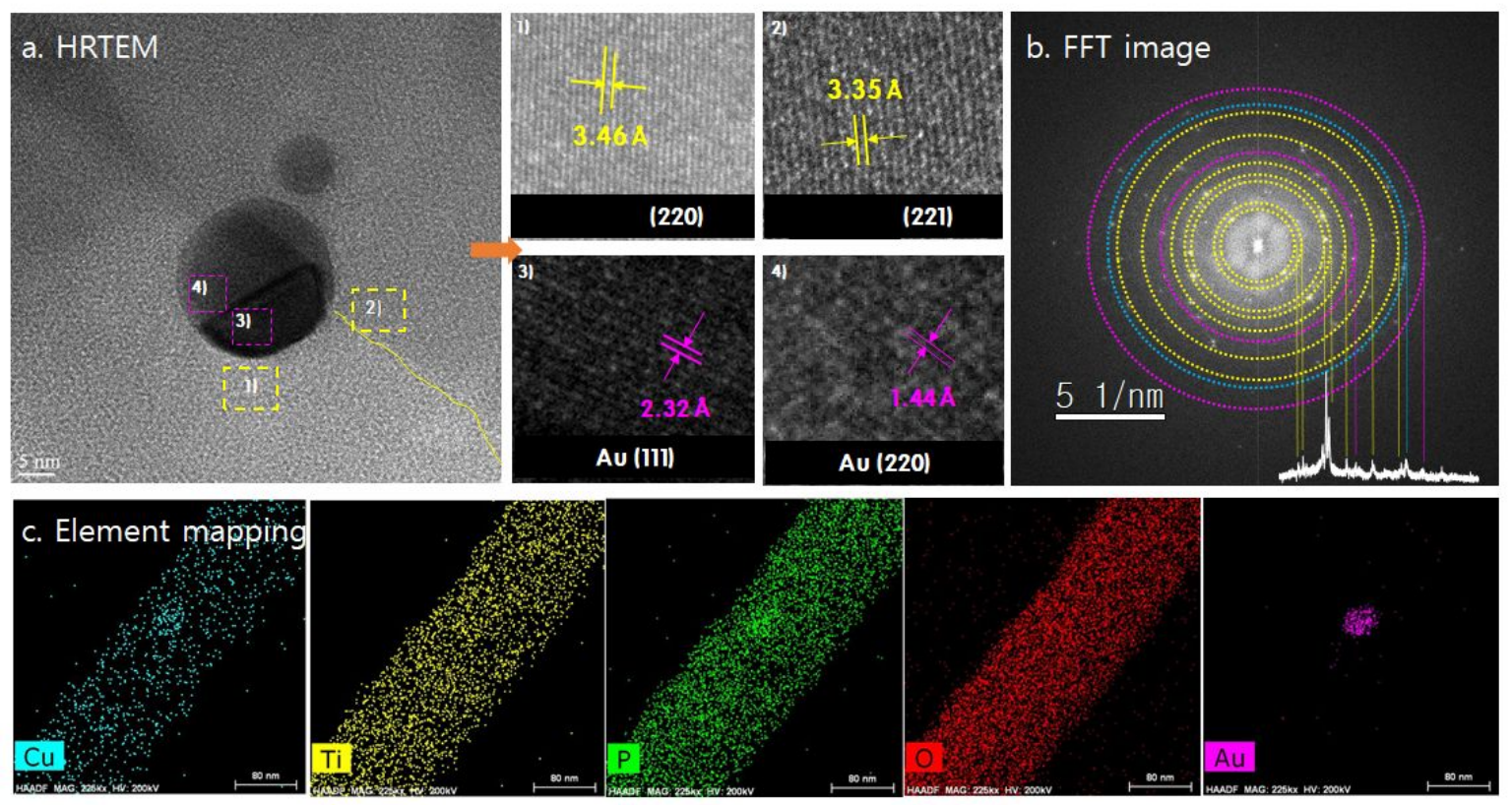

B) CuTiP/Au/CuTiP
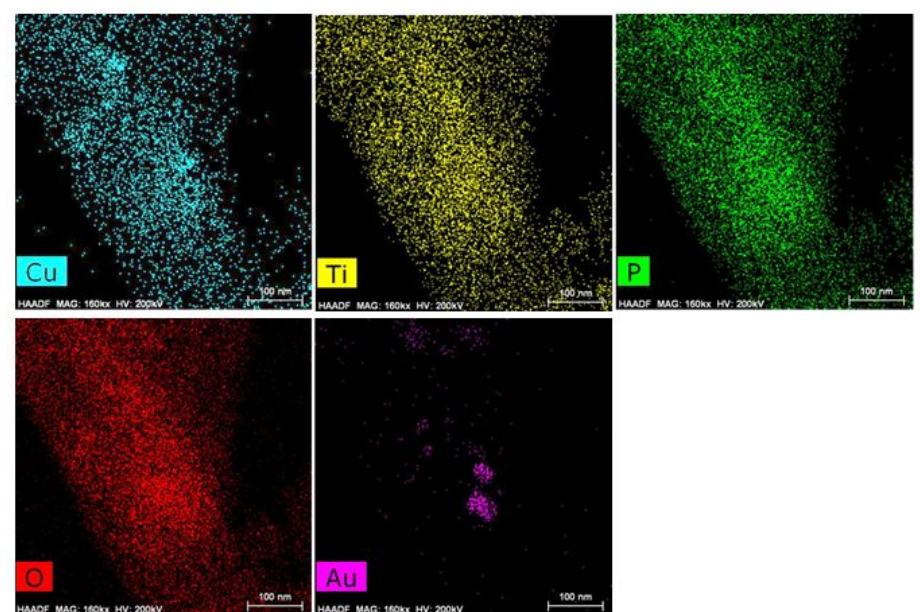

C) Expected CuTiP/Au/CuTiP shape

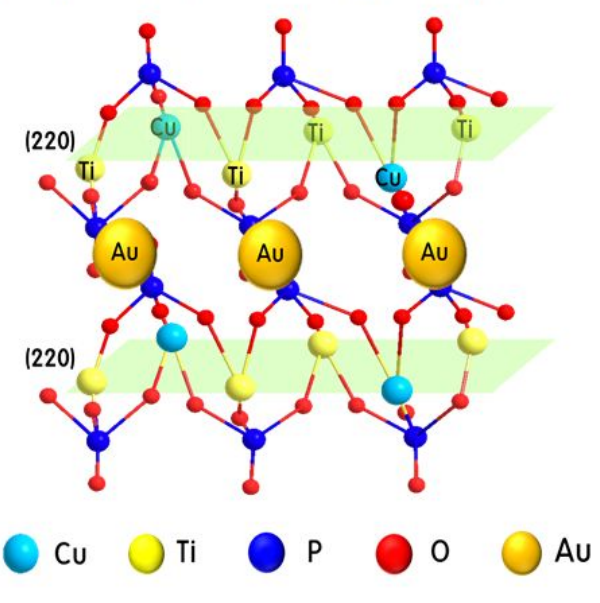

Figure S4. HRTEM analysis for A) CuTiP/Au and B) CuTiP/Au/CuTiP catalysts and C) Expected CuTiP/Au/CuTiP shape. 


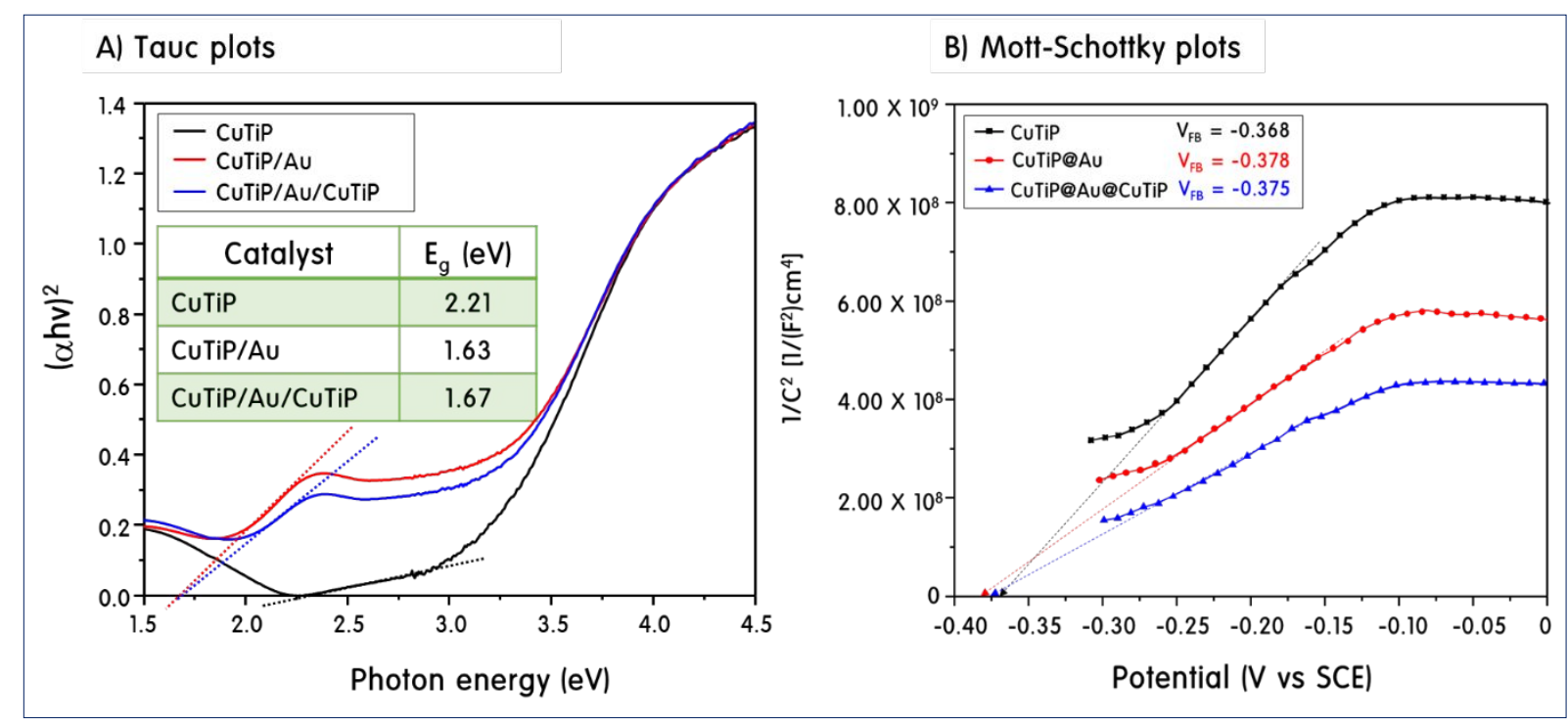

Figure S5. Tauc's plots (A) and Mott-Schottky plots (B) of CuTiP, CuTiP/Au, and CuTiP/Au/CuTiP catalysts. 
a)

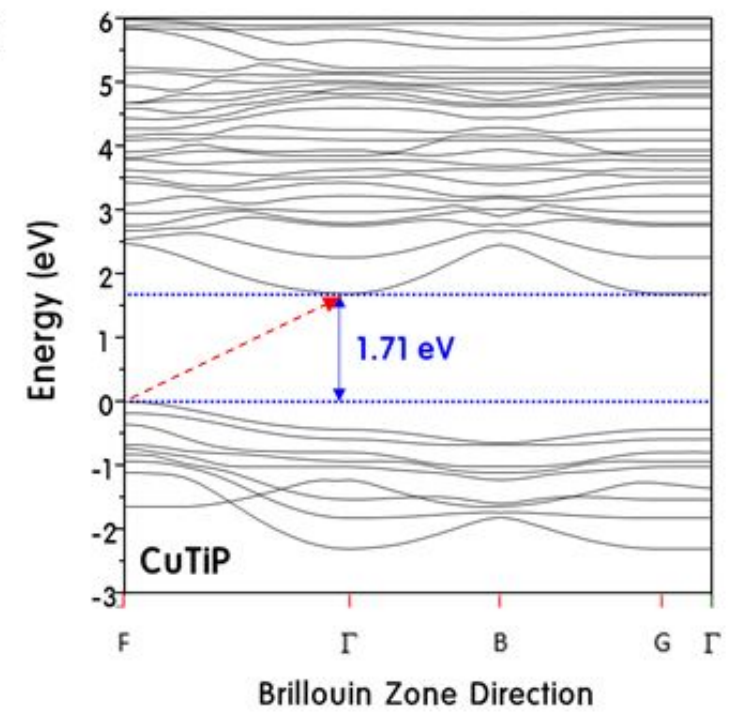

b)

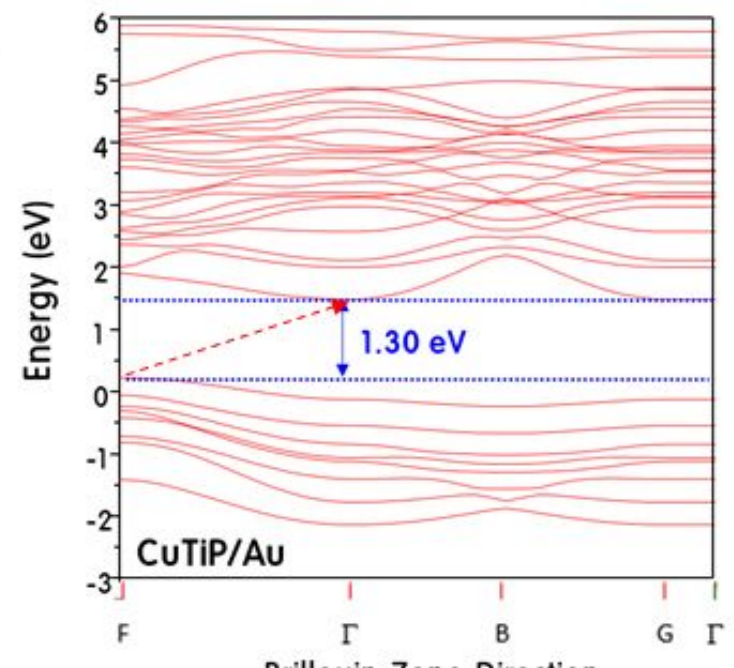

Brillouin Zone Direction
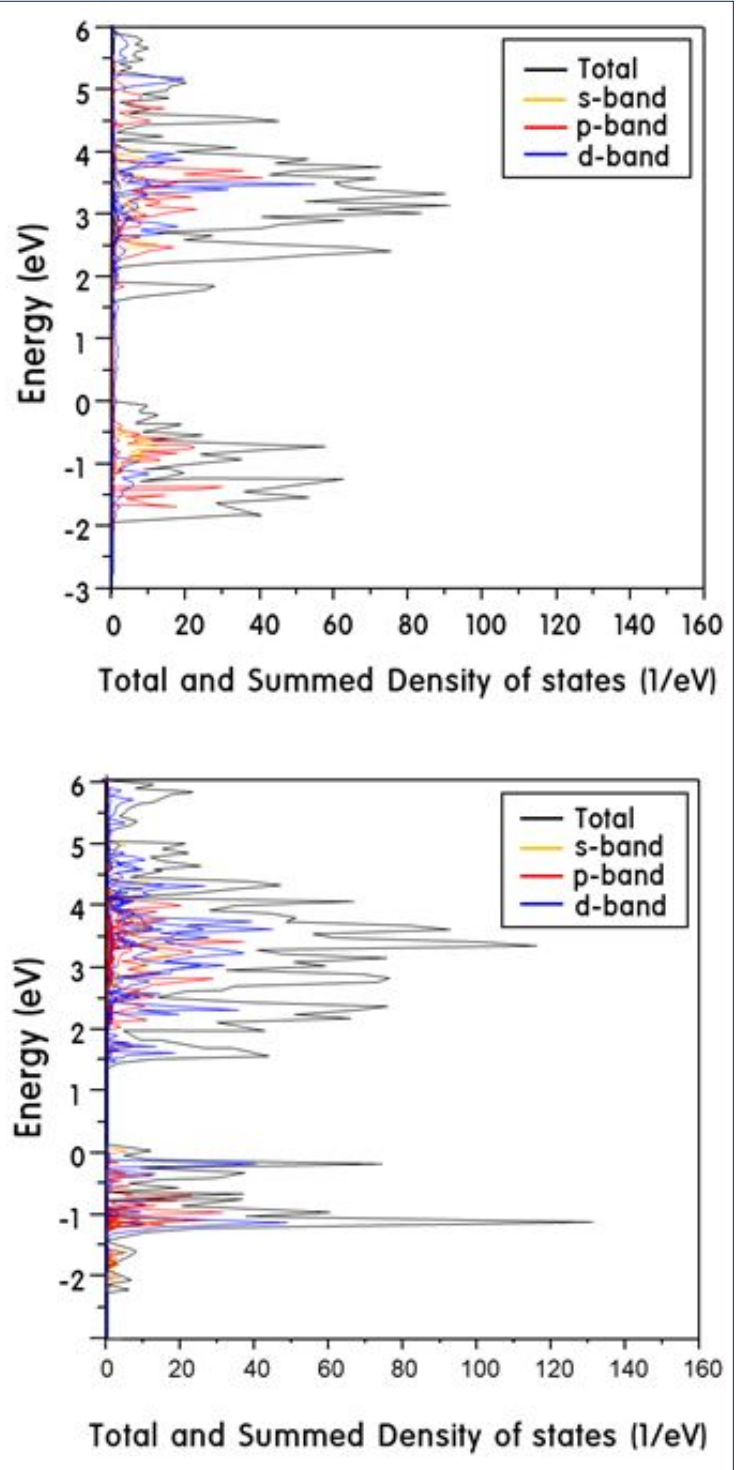

Figure S6. Electronic band structure and density of states (DOS) for a) CuTiP and b) CuTiP/Au catalysts. 

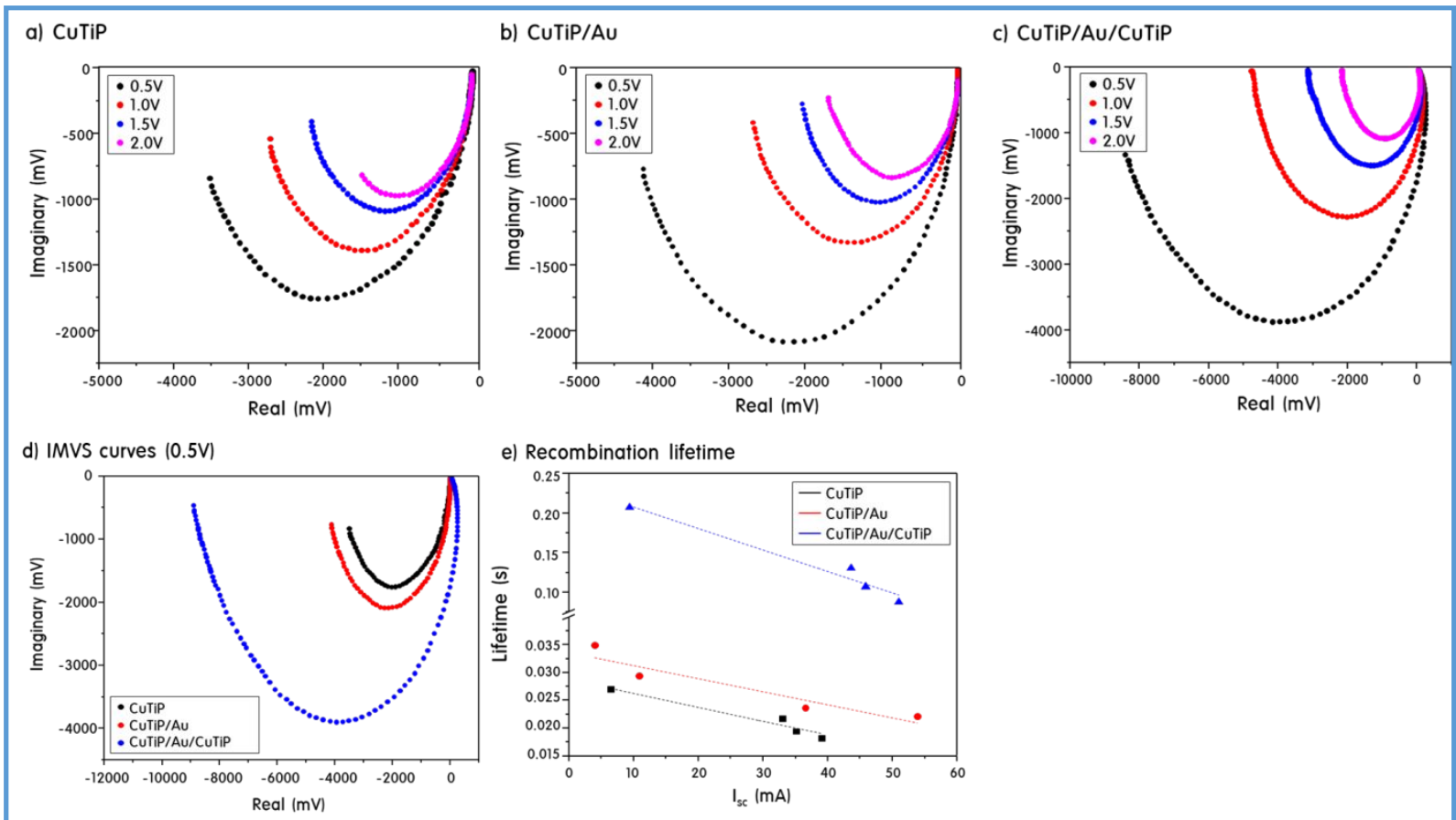

Figure S7. IMVS circles recorded according to the voltage value for the a) CuTiP, b) $\mathrm{CuTiP} / \mathrm{Au}$, and c) $\mathrm{CuTiP} / \mathrm{Au} / \mathrm{CuTiP}$ catalysts, d) comparison of IMVS curves at $0.5 \mathrm{~V}$ and e) calculated recombination lifetime. 


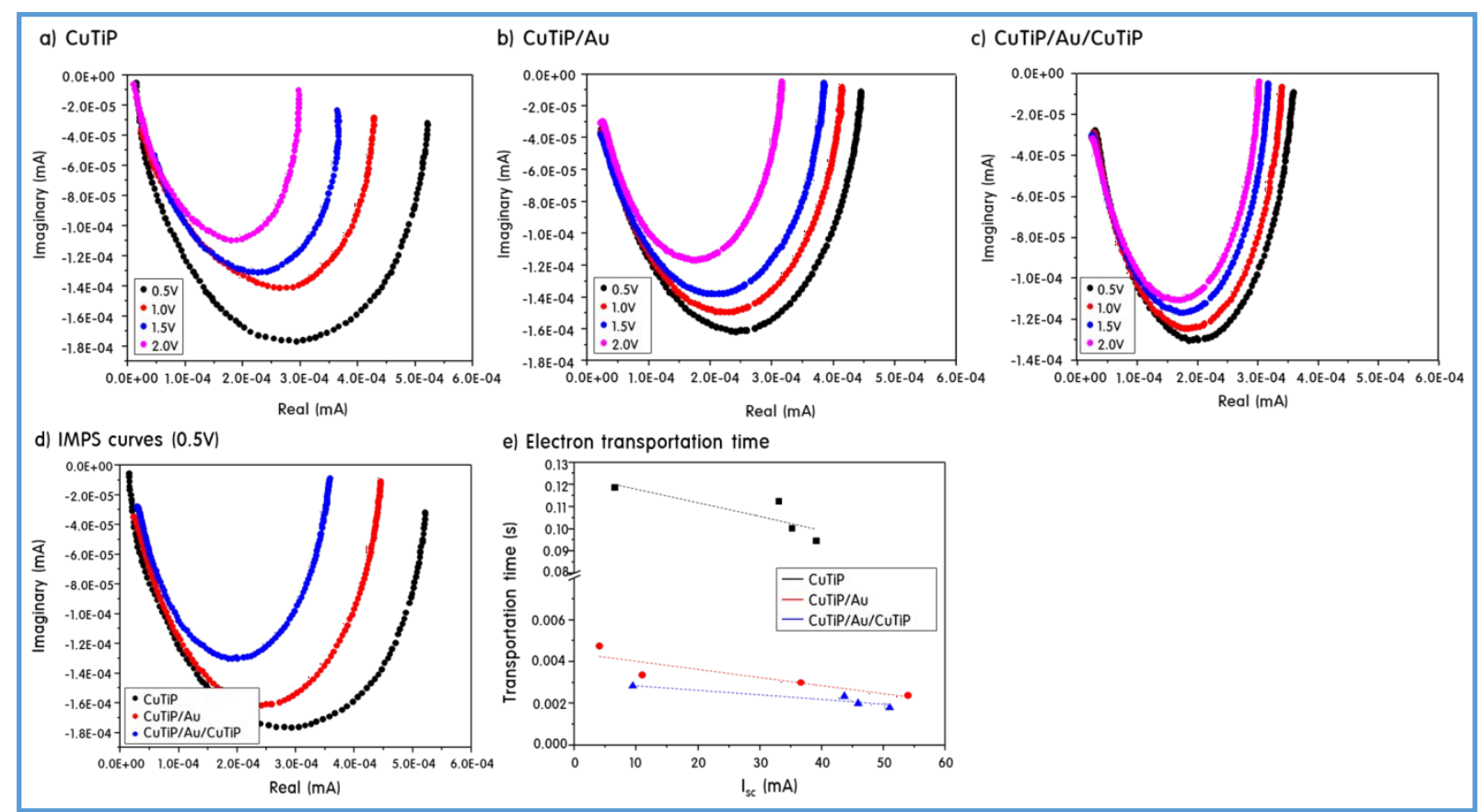

Figure S8. IMPS circles recorded according to the voltage value for the a) CuTiP, b) CuTiP/Au, and c) $\mathrm{CuTiP} / \mathrm{Au} / \mathrm{CuTiP}$ catalysts, d) comparison of IMPS curves at $0.5 \mathrm{~V}$ and e) calculated electron transportation time. 
Table S2. Comparison of the activity of previously studied catalysts.

\begin{tabular}{|c|c|c|c|c|c|c|}
\hline & Catalyst & Light source & Product & Reaction time (h) & Yield of $\mathrm{CH}_{4}\left(\mu \mathrm{molg}^{-1}\right)$ & Ref. \\
\hline \multirow{2}{*}{1} & $\mathrm{TiO}_{2}$ & \multirow{2}{*}{$200 \mathrm{~W}$ Hg reflector lamp } & \multirow{2}{*}{$\mathrm{CO}, \mathrm{CH}_{4}$} & \multirow{2}{*}{10} & $\approx 1.99$ & \multirow[t]{2}{*}{1} \\
\hline & $\mathrm{Cu} / \mathrm{TiO}_{2}$ & & & & $\approx 4.00$ & \\
\hline 2 & $\mathrm{Ag}_{3} \mathrm{PO}_{4} / \mathrm{g}-\mathrm{C}_{3} \mathrm{~N}_{4}$ & $500 W$ Xe lamp & $\mathrm{CO}, \mathrm{CH}_{3} \mathrm{OH}, \mathrm{CH} 4$ & 4 & $\approx 44.85 \mu \mathrm{mol} \mathrm{g}^{-1} \mathrm{~h}^{-1}$ & 2 \\
\hline \multirow{2}{*}{3} & $\mathrm{LaPO}_{4}$ & \multirow{2}{*}{ 125W Hg lamp } & \multirow{2}{*}{$\mathrm{CH}_{4}, \mathrm{H}_{2}$} & \multirow{2}{*}{5} & $0.51 \mu \mathrm{mol}$ & \multirow[t]{2}{*}{3} \\
\hline & $\mathrm{LaPO}_{4}-\mathrm{lwt} \% \mathrm{pt}$ & & & & $3.24 \mu \mathrm{mol}$ & \\
\hline 4 & $\mathrm{LaPO}_{4} / \mathrm{g}-\mathrm{C}_{3} \mathrm{~N}_{4}$ & $300 W$ Xe lamp & $\mathrm{CO}$ & 8 & - & 4 \\
\hline 5 & $\mathrm{Bi}_{2} \mathrm{WO}_{6} / \mathrm{Au}-\mathrm{CdS}$ & $300 W$ Xe lamp & $\mathrm{CH}_{4}$ & 8 & 2.02 & 5 \\
\hline 6 & $\mathrm{Au} / \mathrm{Pt} / \mathrm{TiO}_{2}$ & $500 W$ Xe lamp & $\mathrm{CO}, \mathrm{CH}_{4}$ & 3 & $\approx 1.75 \mu \mathrm{mol}$ & 6 \\
\hline 7 & $\begin{array}{c}2 \mathrm{D}-2 \mathrm{D} \\
\mathrm{MnO}_{2} / \mathrm{g}-\mathrm{C}_{3} \mathrm{~N}_{4}\end{array}$ & 300W Xe lamp & $\mathrm{CO}$ & 6 & - & 7 \\
\hline
\end{tabular}




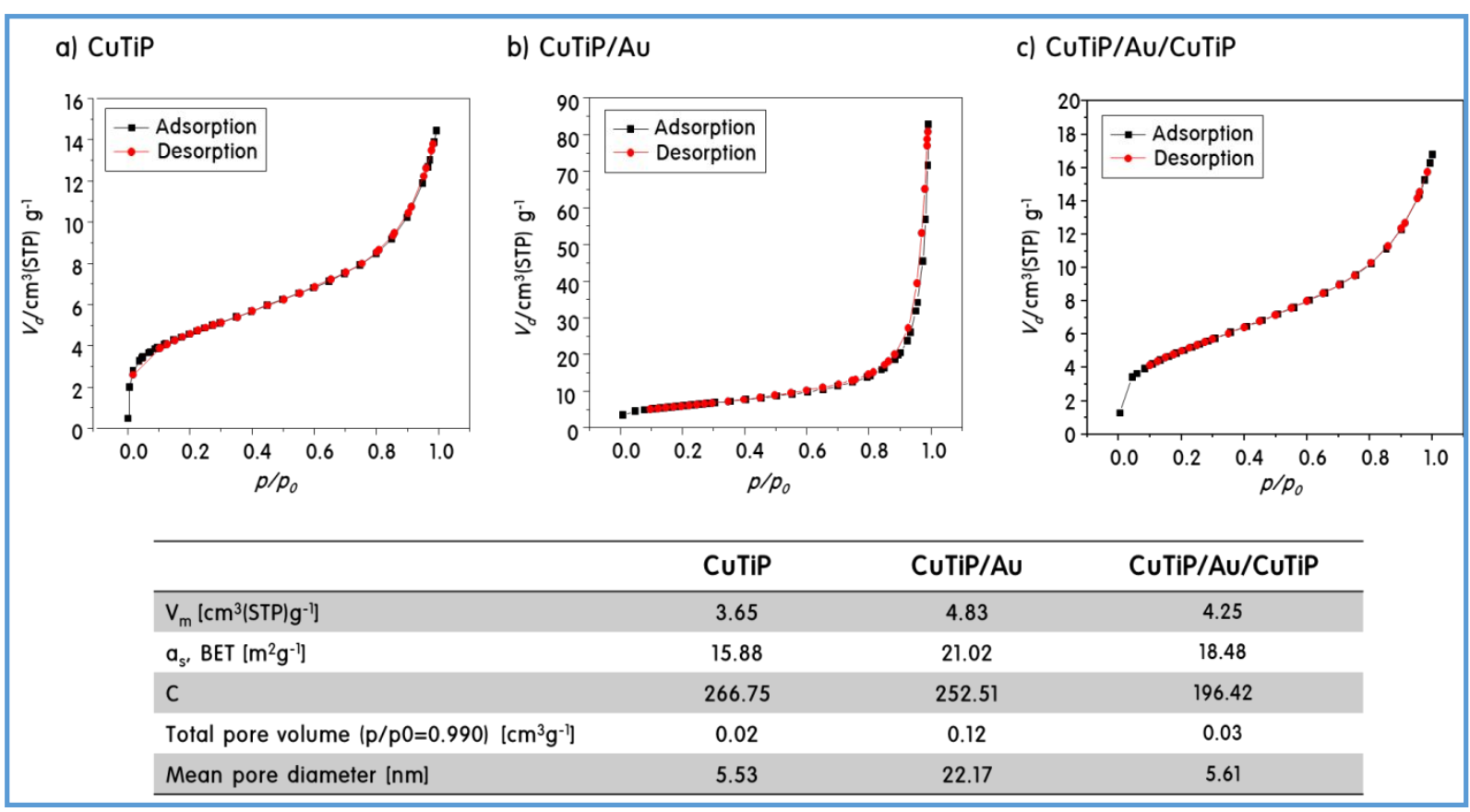

Figure S9. $\mathrm{N}_{2}$ adsorption-desorption isotherm curves for the a) CuTiP, b) CuTiP/Au, and c) CuTiP/Au/CuTiP catalysts.. 


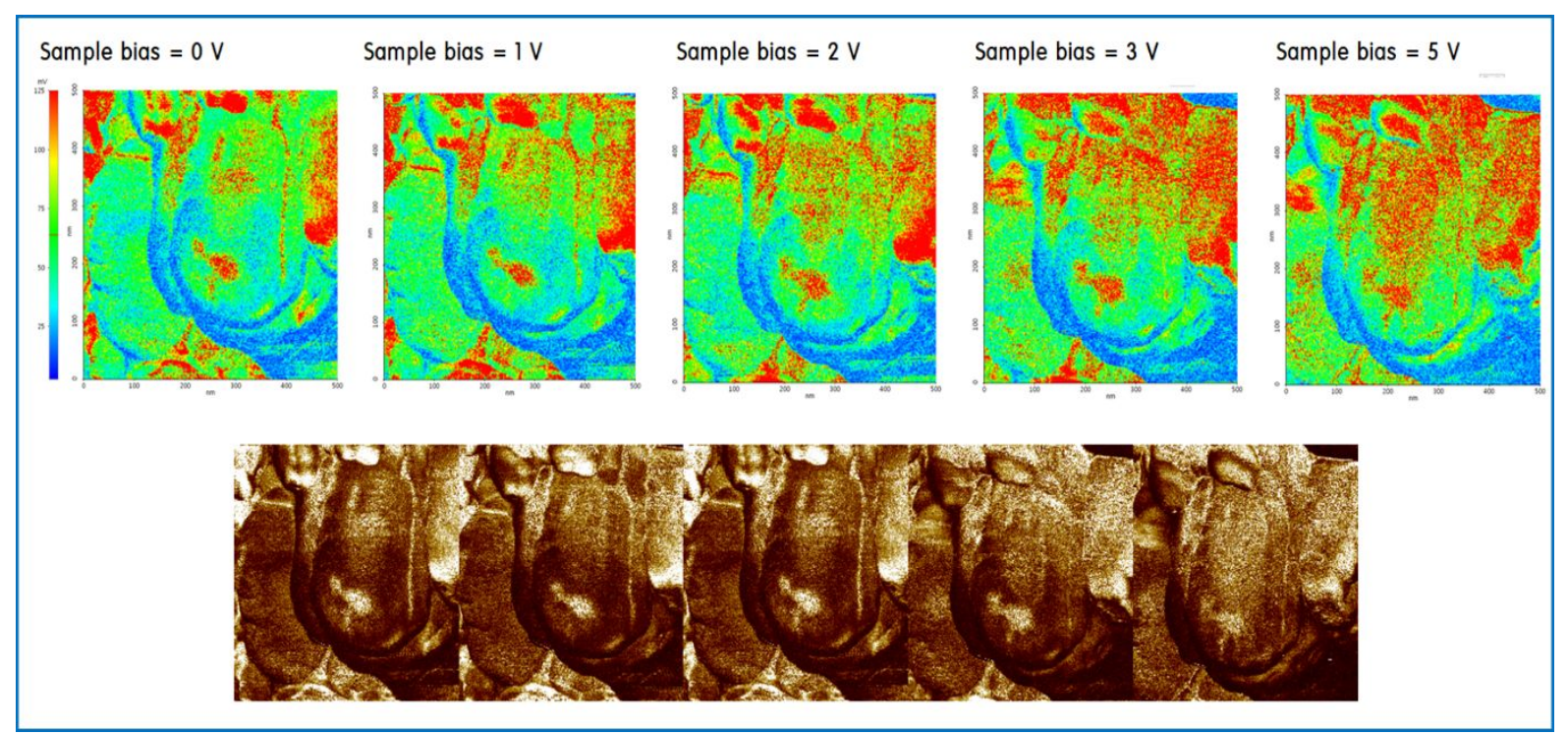

Figure S10. EFM images with voltage change for the CuTiP/Au/CuTiP catalyst. 


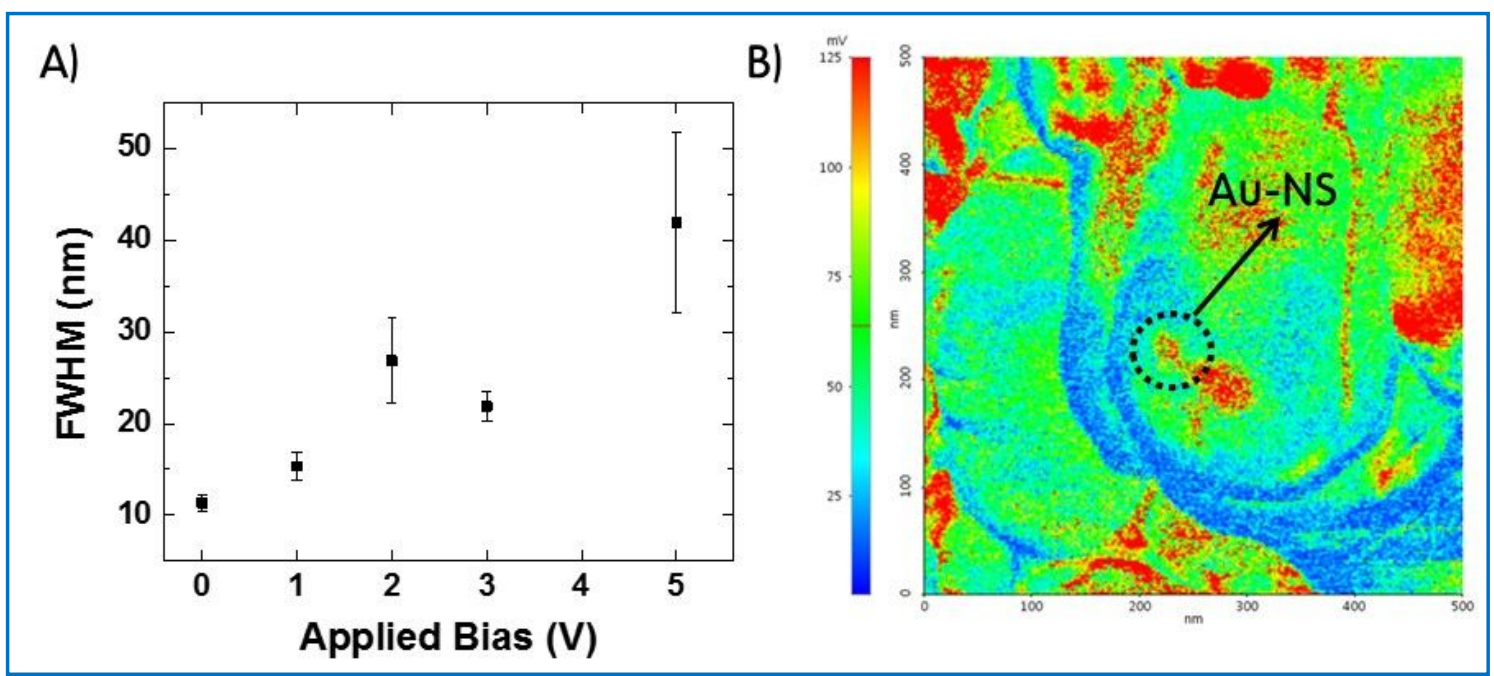

Figure S11. (A) Full width at half maximum of selected potential distribution at Au nanoparticles as marked at (B) 

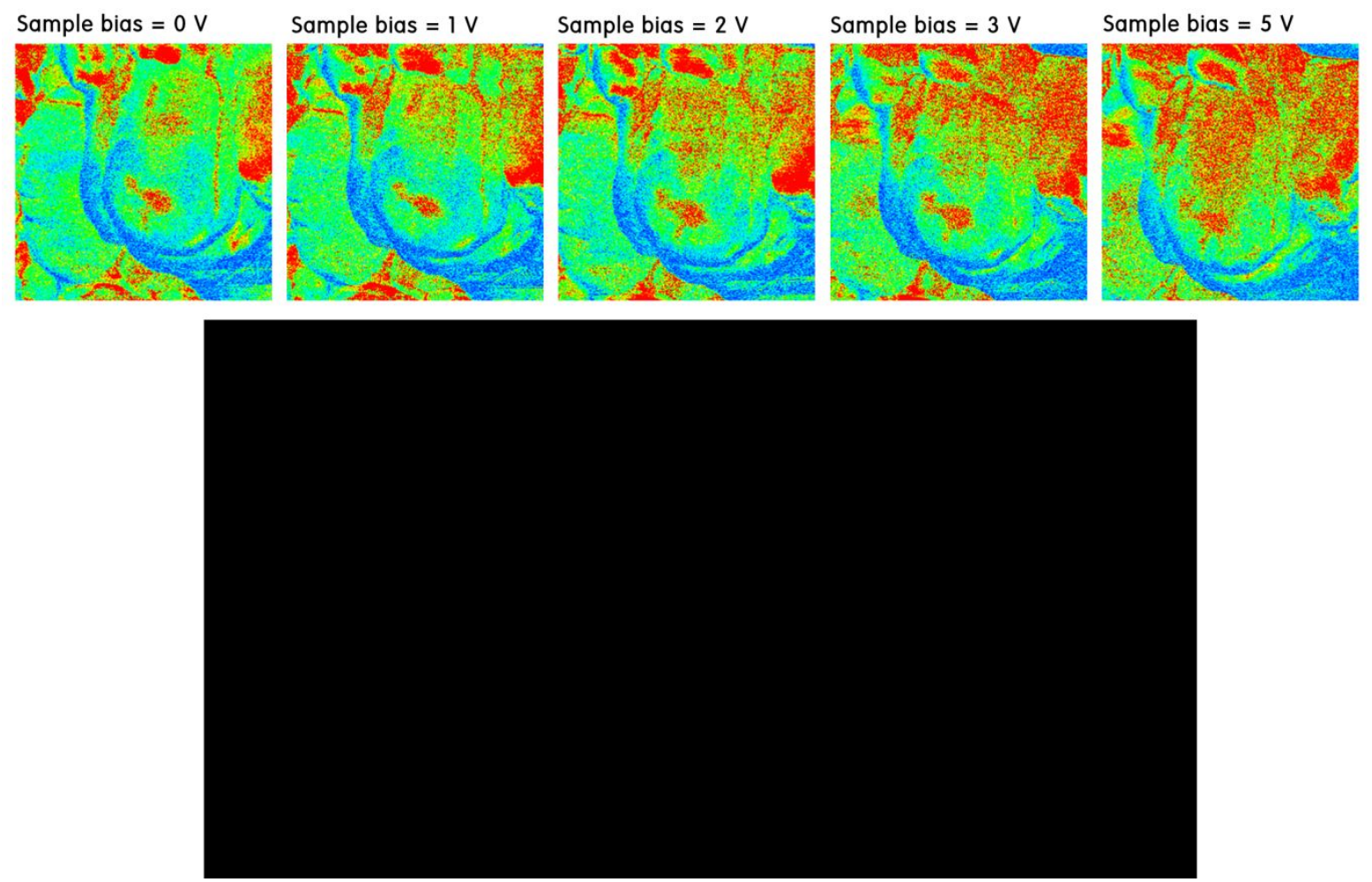

Movie S1. EFM image change video depending on voltage change for the CuTiP/Au/CuTiP catalyst. (Presented separately as a avi file) 
Table S3. DFT calculated electronic energies.

\begin{tabular}{ccc}
\hline Adsorbate & EDFT/eV & EZPE/eV \\
\hline $\mathrm{CO}_{2}$ & -17.618 & -21.142 \\
$\mathrm{CO}$ & -11.396 & -13.675 \\
$\mathrm{CH}_{4}$ & -23.997 & -28.796 \\
$\mathrm{H}_{2} \mathrm{O}$ & -13.786 & -16.543 \\
$\mathrm{H}_{2}$ & -6.759 & -8.111 \\
\hline Substrate & EDFT/eV & \\
\hline $\mathrm{CuTiP}$ & -321.198 & EZPE/eV \\
\hline $\mathrm{CuTiP}-\mathrm{Au}$ & -343.752 & -385.438 \\
\hline $\mathrm{Au}(111)$ & -83.339 & -412.502 \\
\hline $\mathrm{Au}(220)$ & -95.967 & -100.007 \\
\hline
\end{tabular}

$$
E_{\text {ads }}=E_{\text {substrate }+ \text { adsorbate }}-\left|E_{\text {substrate }}+E_{\text {adsorbate }}\right|
$$

\begin{tabular}{|c|c|c|}
\hline$E_{s u b+a d s}$ & $\mathrm{EDFT} / \mathrm{eV}$ & EZPE/eV \\
\hline CUTiP- $\mathrm{CO}_{2}$ & -303.680 & -364.416 \\
\hline CuTiP-CO & -284.577 & -341.492 \\
\hline CUTiP-CH ${ }_{4}$ & -404.612 & -485.534 \\
\hline CUTiP- $\mathrm{H}_{2} \mathrm{O}$ & -340.348 & -408.418 \\
\hline CUTiP- ${ }_{2}$ & -320.429 & -384.515 \\
\hline$E_{s u b+a d s}$ & EDFT/eV & EZPE/eV \\
\hline CuTiPAu- $\mathrm{CO}_{2}$ & -361.5655 & -376.2033 \\
\hline CUTIPAU-CO & -355.7537 & -369.0884 \\
\hline CuTiPAu- $\mathrm{CH}_{4}$ & -367.3032 & -377.5312 \\
\hline CuTiPAu- $\mathrm{H}_{2} \mathrm{O}$ & -357.6467 & -367.7495 \\
\hline CUTIPAU- ${ }_{2}$ & -350.1304 & -360.4542 \\
\hline$E_{s u b+a d s}$ & EDFT/eV & EZPE/eV \\
\hline $\mathrm{Au}(111)-\mathrm{CO}_{2}$ & -100.9103 & -108.9236 \\
\hline $\mathrm{Au}(111)-\mathrm{CO}$ & -94.7872 & -99.4847 \\
\hline $\mathrm{Au}(111)-\mathrm{CH}_{4}$ & -106.3907 & -110.9043 \\
\hline $\mathrm{Au}(111)-\mathrm{H}_{2} \mathrm{O}$ & -97.1971 & -100.9708 \\
\hline $\mathrm{Au}(111)-\mathrm{H}_{2}$ & -89.2930 & -93.9704 \\
\hline$E_{s u b+a d s}$ & EDFT/eV & EZPE/eV \\
\hline $\mathrm{Au}(220)-\mathrm{CO}_{2}$ & -113.7099 & -120.8865 \\
\hline $\mathrm{Au}(220)-\mathrm{CO}$ & -107.4596 & -112.6867 \\
\hline $\mathrm{Au}(220)-\mathrm{CH}_{4}$ & -119.9758 & -121.9495 \\
\hline $\mathrm{Au}(220)-\mathrm{H}_{2} \mathrm{O}$ & -109.8524 & -112.2687 \\
\hline $\mathrm{Au}(220)-\mathrm{H}_{2}$ & -102.3522 & -104.6793 \\
\hline
\end{tabular}




\section{References of Supporting Information}

1. Tahir, M.; Tahir, B. Dynamic Photocatalytic Reduction of $\mathrm{CO}_{2}$ to $\mathrm{CO}$ in a Honeycombmonolith Reactor Loaded with $\mathrm{Cu}$ and $\mathrm{N}$ doped $\mathrm{TiO}_{2}$ Nanocatalysts. Appl. Surf. Sci. 2016, 377, 244-252, DOI 10.1016/j.apsusc.2016.03.141.

2. He, Y.; Zhang, L.; Teng, B.; Fan, M. New Application of Z-scheme $\mathrm{g}_{3} \mathrm{PO}_{4} / \mathrm{g}-\mathrm{C}_{3} \mathrm{~N}_{4}$ Composite in Converting $\mathrm{CO}_{2}$ to Fuel. Environ. Sci. Technol. 2015, 49(1), 649-656, DOI 10.1021/es5046309.

3. Pan, B.; Luo, S.; Su, W.; Wang, X. Photocatalytic $\mathrm{CO}_{2}$ Reduction with $\mathrm{H}_{2} \mathrm{O}$ over $\mathrm{LaPO}_{4} \mathrm{Nanorods}$ Deposited with Pt Cocatalyst. Appl. Catal. B. 2015, 168, 458-464, DOI 10.1016/j.apcatb.2014.12.046.

4. Li, M.; Zhanga, L.; Fan, X.; Wu, M.; Wang, M.; Cheng, R.; Zhang, L.; Yao, H.; Shia, J. Core-shell LaPO $/$ g$\mathrm{C}_{3} \mathrm{~N}_{4}$ Nanowires for Highly Active and Selective $\mathrm{CO}_{2}$ Reduction. Appl. Catal. B. 2017, 201, 629-635, DOI 10.1016/j.apcatb.2016.09.004.

5. Wang, M.; Han, Q.; Li, L.; Tang, L.; Li, H.; Zhou, Y.; Zou, Z. Construction of an All-solid-State Artificial ZScheme System Consisting of $\mathrm{Bi}_{2} \mathrm{WO}_{6} / \mathrm{Au} / \mathrm{CdS}$ Nanostructure for Photocatalytic $\mathrm{CO}_{2}$ Reduction into Renewable Hydrocarbon Fuel. Nanotechnol. 2017, 28, 274002, DOI 10.1088/1361-6528/aa6bb5.

6. Zhang, Z.; Wang, Z.; Cao, S.-W.; Xue, C. Au/Pt Nanoparticle-decorated $\mathrm{TiO}_{2}$ Nanofibers with PlasmonEnhanced Photocatalytic Activities for Solar-to-Fuel Conversion. J. Phys. Chem. C. 2013, 117, 25939-25947, DOI 10.1021/jp409311x.

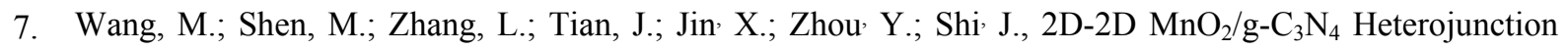
Photocatalyst: In-situ Synthesis and Enhanced $\mathrm{CO}_{2}$ Reduction Activity. Carbon 2017, 120, 23-31, DOI 10.1016/j.carbon.2017.05.024. 